\title{
Development and implementation of Persian test of Elderly for Assessment of Cognition and Executive function (PEACE)
}

\author{
Pari Sadat Haji Seyed Javadi ${ }^{1}$, Azadeh Zendehbad ${ }^{2}$, Fatemeh Darabi ${ }^{3}$, Shahrzad Khosravifar ${ }^{4}$, Maryam \\ Noroozian $^{5}$
}

\footnotetext{
${ }^{1}$ M.D., Resident of Psychiatry, Department of Psychiatry, Tehran University of Medical Sciences, Tehran, Iran.

${ }^{2}$ M.D., Resident of Geriatric Medicine, Department of Geriatric Medicine, Tehran University of Medical Sciences, Tehran, Iran

${ }^{3}$ Ph.D. Candidate of Health Education \& Health Promotion, Department of Public Health, School of Health, Gonabad University of Medical Sciences, Gonabad, Iran

${ }^{4}$ M.D., Resident of Psychiatry, Department of Psychiatry, Iran University of Medical Sciences, Tehran, Iran

${ }^{5}$ M.D., Professor of Neurology, Director, Memory and Behavioral Neurology Division, Tehran University of Medical Sciences, Iran
}

Type of article: Original

\begin{abstract}
Introduction: A considerable segment of the elderly population in Iran is illiterate, and it seems the existing neuropsychological screening tests are not very useful for detecting dementia in illiterate participants. The purpose of this study was to develop and validate a tool called Persian test of Elderly for Assessment of Cognition and Executive function (PEACE) for detecting dementia in both illiterate and literate participants.

Methods: First, in order to design some of the cognitive aspects of the PEACE assay, we considered other prevalent neuropsychological instruments, such as the General Practitioner assessment of Cognition (GPCOG), Functional Assessment Staging (FAST), Mini Mental State Examination (MMSE), and Wechsler Memory scale. The other domains of PEACE were designed according to our clinical proficiencies and the culture of the society. In the next step, the participants were classified into three distinct groups, i.e., the control group ( $\mathrm{n}=33$ ), the Mild Cognitive Impairment (MCI) group $(n=30)$, and the Alzheimer's group $(n=38)$. All of the participants in each group were divided according to their educational level, i.e., illiterate, semi-literate, and literate.

Results: We developed PEACE consisting of 14 items, each of which represents a specific cognitive function, with a maximum score of 91 . The 14 items are Orientation, Praxis, Attention and Concentration, Attention and Calculation, Memory, Similarity, Abstract Thinking, General Information, Language, Judgment, Gnosis, Planning (Sequencing), Problem Solving, and Animal Naming. PEACE scores are highly correlated with those of the MMSE ( $\mathrm{r}=0.78)$. The optimal cut-off point of PEACE chosen for diagnosis of Alzheimer's disease was 67.5 (sensitivity: 75.8\%, specificity: 97.4\%). The PEACE scores showed a significant difference between Participants with Alzheimer's disease and the control group $(\mathrm{p}=0.0000)$ and the MCI group $(\mathrm{p}=0.003)$. In addition, there was no significant difference between illiterate and literate participants in the Alzheimer's group. However, the PEACE scores differed significantly $(\mathrm{p}=0.0000)$ between illiterate and literate participants in the control group.

Conclusion: The PEACE addresses the limitations of existing tests and is appropriate for use in countries that have high rates of illiteracy. It is a valid screening mechanism for the detection of dementia in both illiterate and literate participants.
\end{abstract}

Keywords: Alzheimer's, literate, semi-literate, illiterate, PEACE assay

\section{Corresponding author:}

Dr. Pari Sadat Haji Seyed Javadi, Roozbeh Hospital, Department of Psychiatry, Tehran University of Medical Sciences, Tehran, Iran. Tel: +98.2164351, Fax:+98.64352215, Email: P.Sedjavadi@gmail.com

Received: July 10, 2015, Accepted: September 04, 2015, Published: November 2015

iThenticate screening: September 03, 2015, English editing: September 17, 2015, Quality control: November 05, 2015

(C) 2015 The Authors. This is an open access article under the terms of the Creative Commons Attribution-NonCommercialNoDerivs License, which permits use and distribution in any medium, provided the original work is properly cited, the use is non-commercial and no modifications or adaptations are made. 


\section{Introduction}

Presently, population aging is occurring as a global phenomenon. In most nations, the fastest-growing age group is 80 and over (1). In Iran, $8.2 \%$ of the population is over 65 (2). Research related to the oldest members of the population poses serious challenges because of the prevalence of health problems and sensory impairments. The issues include functional disability, frailty, and fatigue, all of which make the assessment of cognitive, occupational, and social functioning problematic (3). Advancing age is the most significant risk factor for dementia (4). Dementia is associated with increased mortality in institutionalized older adults. The prevalence of dementia in industrialized countries has been estimated to be in the range of 4 to $8 \%$ among people over 65 (5). The most common cause of dementia in elderly people is Alzheimer's disease (AD), which contributes to about 60 to $70 \%$ of all cases of dementia (1). Alzheimer's disease is a neurodegenerative disorder that causes deterioration of cognitive functions, including memory, language, agnosia, attention, apraxia, executive functions, and orientation (6). This catastrophic illness has resulted in significant increases in the cost of healthcare (7). Current evidence shows that there is an inverse association between educational level and the risk of $\mathrm{AD}$ or dementia $(1,8)$.

Early detection and screening have become accepted approaches for identifying dementia (9). However, most neuropsychological screening tests often are developed for the literate population and, therefore, include literacydependent items $(6,10)$. A considerable segment of the elderly population in Iran is illiterate, and very few people have a formal education. Many of these people are able to read, but they cannot write. Generally, $97 \%$ of rural women have had little or no education due to the fact that education has not been considered to be use ful for women in their later life $(11,12)$. This illiteracy in Iran will continue at least for the next 10 to 30 years or longer, so it seems that the available neuropsychological screening tests will not be useful for detecting dementia in a large segment of the population. Thus, there is a pressing need to develop tools that can be used to assess dementia in illiterate elderly people $(13,14)$. The first author has 15 years of professional experience with cognitive disorders, including dementia among Iran's elderly population of which more than $70 \%$ are illiterate. In this experience, many shortcomings have been observed, including defective measurement tools that provide unreliable diagnoses of cognitive impairment in illiterate people or people who have low levels of education. Some of the measurement tools have been found to be defective even when used to assess educated people who have different cultures and life routines (15). Thus, we developed the PEACE assay with the aim of detecting different cognitive aspects in both illiterate and literate elderly people. Other aims of this study were to validate PEACE for Iran's elderly people and to develop cut-off points for PEACE so that it can be used to identify those who have Alzheimer's disease and those who do not. This questionnaire might be applicable to Iran's neighboring countries, eastern Mediterranean countries, and possibly other developing countries throughout the world.

\section{Material and Methods}

\subsection{PEACE Assay}

This neuropsychological instrument was proposed to assess the cognitive efficiency of both illiterate and literate elderly people. In order to design some of the cognitive aspects of this assay, we considered other prevalent neuropsychological instruments, such as the General Practitioner assessment of Cognition (GPCOG), Functional Assessment Staging (FAST), Mini Mental State Examination (MMSE), and the Wechsler Memory scale. . This scale has been developed by Maryam Noroozian, Professor of Neurology who is an expert in dementia in Iran. Based on her experiences on the impact of illiteracy on the routine assessment scales especially in developing societies like Iran, most of the scales like ADAS COG, MoCA, MMSE and even Clock Drawing Test (CDT) may induce false positive results in uneducated or law-educated subjects. Moreover, some of the other domains and subtests of PEACE were based on the clinical proficiencies of one of the authors and the culture of the society. Actually, PEACE is a Persian questionnaire that was developed from a mixture of prevalent neuropsychological questionnaires and Iranian culture. Before starting a validation study, the administration of the PEACE was determined in a separate pilot study, and its scores were rectified.

PEACE was presented in Persian and there was no time limitation for the assay. In a silent environment, this questionnaire was completed by a trained interviewer who was not informed about the groups. The questionnaire consists of 14 items, each representing a specific cognitive function, i.e., Orientation, Praxis, Attention and Concentration, Attention and Calculation, Memory, Similarity, Abstract Thinking, General Information, Language, Judgment, Gnosis, Planning (Sequencing), Problem Solving, and Animal Naming. 


\subsection{MMSE Assay}

The MMSE is a neurocognitive test designed to screen cognitive impairment. It measures orientation, registration, attention, recall, language, and visuospatial functions. Its scores range from 0 to 30: the higher scores indicate better cognition, and scores below 25 indicate cognitive impairment. It can be administered in 5-10 minutes. The MMSE was validated for use with the elderly Iranian population (16).

\subsection{FAST Assay}

Functional Assessment Staging (FAST) is used to assess the stage of dementia of a person with Alzheimer's disease and is designed to evaluate participants at the moderate-to-severe stages of dementia when the MMSE no longer can reflect changes in a clinically meaningful way. It focuses more on an individual's level of functioning and the activities of daily living versus cognitive decline, and it has seven stages (16).

\subsection{Participants}

The study protocol was approved by the Research Ethics Committee of Tehran University of Medical Sciences, and all participants and their companions were given explanations of the research before it began. All participants who freely agreed to participate in the study signed a free and informed consent statement. The World Health Organization (WHO) defines elderly people in developing countries as those whose ages are equal to or greater than 60. In our study, there were 101 participants whose ages were greater than 60, who spoke Persian, and had different educational levels, i.e., from illiterate to literate. The participants were recruited from Private medical centers and governmental Medical Center of Memory Disorders Investigation and interviewed during 2013 and 2014. The exclusion criteria were a known history of neurological and psychological disease, head trauma, stroke, epilepsy, or any other physical illness that affected cognitive functions; visual or hearing difficulties that would interfere with the testing procedure; FAST stage above a score of 6; and motor impairment that could affect the test scores. The participants were classified into three distinct groups:

1) Control group: The participants in this group had no cognitive complaints or any history of neurological and psychiatric disorders. They were not taking any medication that might have affected their cognitive status and mood.

2) Mild Cognitive Impairment (MCI) group: These participants had mild cognitive impairment.

3) Alzheimer's group: These participants had dementia that had been diagnosed by a neurologist based on the criteria of the fourth edition of the Diagnostic and Statistical Manual of Mental Disorders (DSM-IV) (17) and according to the criteria established by the National Institute of Neurological and Communicative Disorders and Stroke/Alzheimer's Disease and Related Disorders Association (NINCS-ADRDA) (18).

The participants in each group were divided according to their educational level, as follows: 1) Illiterate (not being able to read and write), 2) Semi-literate (elementary school), and 3) Literate (higher than elementary school). The participants' degree of literacy was based on whether they could read and write. If the cognitive change was due to the progression of Alzheimer's disease and the participants were diagnosed with dementia, the FAST assay was performed by a research assistant, and then we performed PEACE and MMSE assays in all of the groups. The reliability and constancy of the PEACE assay was confirmed by Cronbach's alpha.

\subsection{Statistical analyses}

Statistical significance was determined using SPSS software version 19 (SPSS, Inc., Chicago, IL, USA). To assess the ability of the PEACE assay to screen dementia, separate receiver operating characteristics (ROC) curves were plotted and defined as the scores that led to the maximal accuracy of classification of participants and non-patient participants. To measure the reliability of the test-retest, we assessed the intra-class correlation coefficient (Cronbach's alpha) and the extra-class correlation coefficient for the baseline and the four-week follow-up PEACE scores. For non-parametric data, the f-Wallis test was used, when appropriate, and it was followed by the MannWhitney $U$ test when a significant difference was found. For the parametric data, we used analysis of variance (ANOVA) for multiple comparisons, followed by the post hoc Tukey-Kramertest. A significance level of $\mathrm{p}<0.05$ was used in all cases.

\section{Results}

A value of Cronbach's alpha above 0.7 indicated that the PEACE questionnaire has reliable and dependable. In our study, Cronbach's alpha for the PEACE scores was 0.876 , and the deletion of each question from the questionnaire showed a Cronbach's alpha above 0.7. Test-retest reliability data were collected from 40 participants at four-week 
intervals. The extra-class correlation coefficient between the baseline scores and the follow-up was 0.928 ( $p<$ 0.05).A good positive correlation existed between PEACE and MMSE $(r=0.78 ; \mathrm{p}<0.001)$.

Table 1 presents the age and gender information for each group. This study was conducted on 33 control participants, 30 MCI participants, and 38 Alzheimer's participants. Among the females, 24.75\% had Alzheimer's disease, but only $7.92 \%$ of the males were so afflicted. The comparison of the demographic variables for age showed a significant difference $(\mathrm{p}=0.001)$ between Participants with Alzheimer's disease $(74.60 \pm 8.02)$ and control participants $(67.84 \pm 7.29)$; also, there was a significant difference $(\mathrm{p}=0.034)$ between the MCI group $(72.5 \pm 7.2)$ and the control group. There was no difference between the ages of the MCI and Alzheimer's groups. The mean ages of the illiterate people in the MCI group $(73.5 \pm 8.15 ; \mathrm{p}=0.021)$ and in the Alzheimer's group (78.18 \pm 4.51 ; $\mathrm{p}=0.0000)$ were different from that of the illiterate people in the control group $(65.7 \pm 4.52)$. The mean ages of semiliterate Participants with Alzheimer's disease $(77.5 \pm 6.97 ; \mathrm{p}=0.003)$ and of the semi-literate participants in the MCI group $(76 \pm 2 ; \mathrm{p}=0.01)$ were greater than the mean age of the semi-illiterate participants in the control group (65.16 \pm 4.83).In this regard, there was no significant difference between the literate participants in any of the groups. Table 1 gives the mean scores of the PEACE and MMSE tests. In order to examine the main effects and the interactive effects of the groups on the PEACE and the MMSE scores, multi-way ANOVA was conducted. For the MMSE scores, the analysis of variance (ANOVA) showed that there was a significant difference $(\mathrm{p}=0.0000)$ between Participants with Alzheimer's disease $(16.93 \pm 6.03)$ with people in the control group $(25.86 \pm 4.49)$ and in the MCI group (23.03 \pm 4.20$)$. All educational levels of Participants with Alzheimer's disease had significant differences $(p<0.05)$ between their MMSE scores and those of the control group. For PEACE scores, this analysis showed that Participants with Alzheimer's disease $(57 \pm 16.51)$ were significantly $(\mathrm{p}=0.003)$ different from the control group $(82.65 \pm 7.15)$ and the MCI group $(75.76 \pm 11.48)$.

Table 1. Mean scores of tests and demographic data

\begin{tabular}{|c|c|c|c|c|c|c|c|c|c|c|c|c|c|}
\hline \multirow{2}{*}{\multicolumn{2}{|c|}{ Variables }} & \multicolumn{4}{|c|}{ Control group } & \multicolumn{4}{|c|}{ MCI group } & \multicolumn{4}{|c|}{ Alzheimer group } \\
\hline & & $\mathrm{A}^{1}$ & $\mathrm{~B}^{2}$ & $\mathrm{C}^{3}$ & Total & A & B & C & Total & A & B & $\mathrm{C}$ & Total \\
\hline \multirow[t]{2}{*}{ Age } & Mean & 65.7 & 65.17 & 69.55 & 67.84 & 73.5 & 76.0 & 71.0 & 72.5 & 78.18 & 77.5 & 71.06 & 74.61 \\
\hline & SD & 4.52 & 4.83 & 8.49 & 7.29 & 8.16 & 2 & 7.57 & 7.21 & 4.51 & 6.98 & 9.02 & 8.02 \\
\hline \multirow[t]{2}{*}{ Gender } & $\mathrm{F} / \mathrm{M}$ & $5 / 5$ & $4 / 2$ & $11 / 11$ & $20 / 18$ & $4 / 4$ & $3 / 2$ & $9 / 8$ & $16 / 14$ & $10 / 1$ & $5 / 1$ & $10 / 6$ & $25 / 8$ \\
\hline & $\%$ & $50 / 50$ & $67 / 33$ & $50 / 50$ & $53 / 47$ & $50 / 50$ & $60 / 40$ & $53 / 47$ & $53 / 47$ & $91 / 9$ & $83 / 17$ & $62 / 38$ & $76 / 24$ \\
\hline \multirow{2}{*}{$\begin{array}{l}\text { PEACE } \\
\text { Score }\end{array}$} & ME $A$ & 72.8 & 83.08 & 87.02 & 82.66 & 60.75 & 72.8 & 83.71 & 75.77 & 53.09 & 54.0 & 60.81 & 57.0 \\
\hline & SD & 6.01 & 4.50 & 1.89 & 7.15 & 6.27 & 8.79 & 4.47 & 11.48 & 12.98 & 19.15 & 17.81 & 16.52 \\
\hline \multirow{2}{*}{$\begin{array}{l}\text { MMSE } \\
\text { Score }\end{array}$} & MEAN & 20.6 & 24.0 & 28.77 & 25.87 & 18.75 & 19.0 & 26.23 & 23.03 & 12.64 & 15.17 & 20.56 & 16.94 \\
\hline & SD & 1.43 & 6.32 & 1.48 & 4.50 & 1.75 & 2.55 & 1.99 & 4.21 & 3.78 & 4.40 & 5.73 & 6.03 \\
\hline
\end{tabular}

1: Illiterate, 2: Semi-literate, 3: Literate

The comparison of PEACE scores for all educational levels in each group showed that there was no difference $(\mathrm{p}=0.172)$ between illiterate $(53.09 \pm 12.98)$ and literate $(60.81 \pm 17.80)$ participants in the Alzheimer's group; in the control group, there was a significant difference $(\mathrm{p}=0.0000)$ between the illiterate participants $(72.8 \pm 6.01)$ and the literate participants $(87.02 \pm 1.89)$. In addition, the literate participants $(83.7 \pm 4.47 ; \mathrm{p}=0.0000)$ in the MCI group had a higher score than the illiterate participants $(60.75 \pm 6.27)$. The mean scores of PEACE for semi-literate participants in the MCI group ( $72.8 \pm 8.78 ; \mathrm{p}=0.031)$ and for semi-literate participants in the Alzheimer's group (54 $\pm 19.15 ; \mathrm{p}=0.0000)$ were different from the score of the semi-literate participants in the control group (83.08 \pm 4.49$)$. The mean score of PEACE in the illiterate Participants with Alzheimer's disease (53.09 $\pm 12.98 ; \mathrm{p}=0.004)$ was less than that for the participants in the control group $(72.8 \pm 6.01)$. In addition, the mean score of PEACE in the literate Participants with Alzheimer's disease $(60.81 \pm 17.80 ; \mathrm{p}=0.0000)$ was significantly different from that of participants in the control group $(87.02 \pm 1.89 ; \mathrm{p}=0.0000)$; however, there was no difference between the mean score of the literate participants in the MCI group $(83.70 \pm 4.47 ; \mathrm{p}=0.56)$ and the mean score of literate participants in the control group.

Analysis of PEACE sub-domains showed that Participants with Alzheimer's disease and participants in the control group were significantly different $(\mathrm{p}<0.05)$; the comparison of all educational levels in each group for these subdomains indicated that the illiterate and literate participants were not significantly different. The mean ages of illiterate participants $(72.58 \pm 7.74)$, semi-literate participants $(72.70 \pm 7.53)$, and literate participants $(70.43 \pm 8.25)$ 
were not different. Females comprised $65.51 \%$ of the illiterate participants, $70.5 \%$ of the semi-literate participants, and $54.54 \%$ of the literate participants. ROC curves were plotted to indicate how well the PEACE subtest scores identified the status of dementia. The cut-off point (Figure1) for detection of Participants with Alzheimer's disease and Participants without Alzheimer's disease was 67.5 (sensitivity: 75.8\%; specificity: 97.4\%; area under the ROC curves (AUC): 0.88 ). This indicates that $2.4 \%$ of non- Alzheimer's participants and $75.8 \%$ of Alzheimer's participants were above the cut-off point; so PEACE can discriminate between Participants with Alzheimer's disease from Participants without Alzheimer's disease in $96.2 \%$ of the cases.

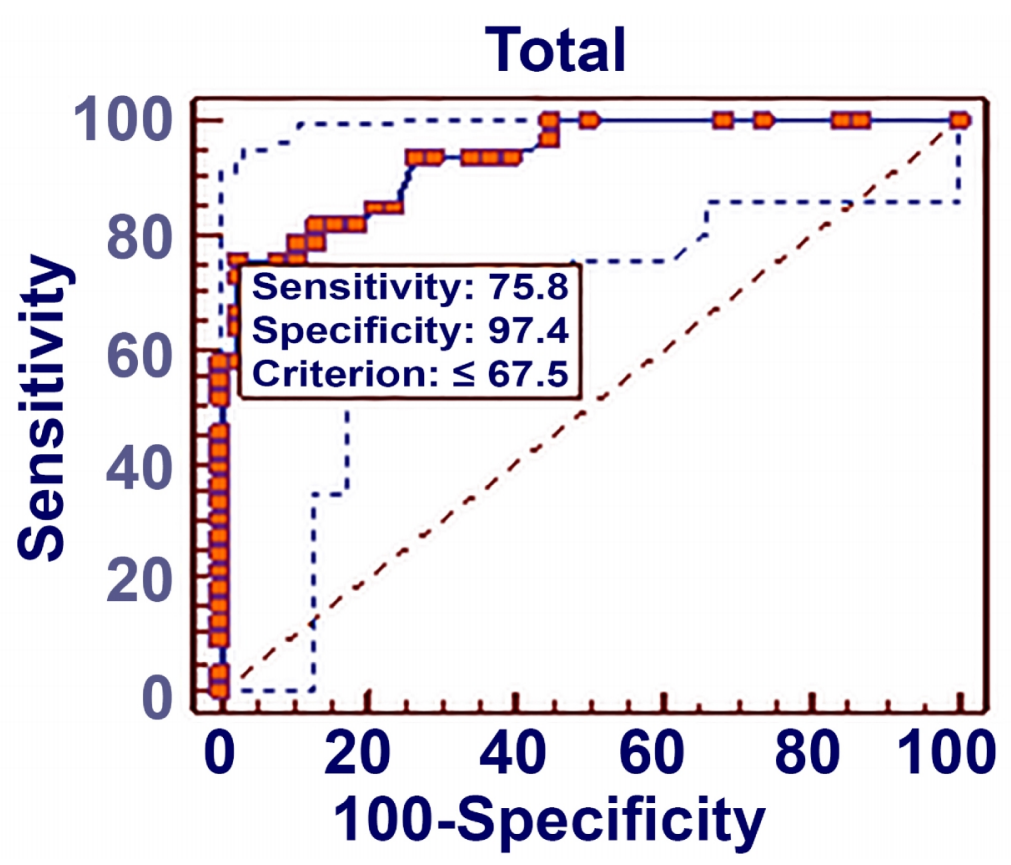

Figure 1. Receiver operating characteristic (ROC) curve analysis of the PEACE scores for differentiating between Participants with Alzheimer's disease and participants without Alzheimer's disease

\section{Discussion}

Advancing age is associated with an overall increased risk for dementia (4), and there is a need for detection and management of this condition (19). Most neuropsychological tests are influenced by age, education, and cultural differences (6).The MMSE, a widely-used dementia screening instrument, is affected strongly by the subject's literacy level and education. Researchers have suggested that countries with low educational levels need instruments tailored to their own environment that can be used to test for cognitive ability (13). In the absence of appropriate neuropsychological batteries for illiterate people, neuropsychological evaluation may present major difficulties, including misdiagnosis and/or delayed detection of dementia in these populations; in addition, the diagnosis often is quite challenging (6). The presence of a huge number of illiterate participants (at least for the next 10 to 30 years) and elderly people in Iran led us to introduce the PEACE test, which can be used for both illiterate and literate participants in assessing cognitive impairments in elderly people. The positive correlation between PEACE and MMSE that was shown in this study indicates that the PEACE test could be used advantageously when accompanied by adequate concurrent validity for the detection of Alzheimer's disease since the cut-off point of this test was 67.5.The results for the PEACE assay in this study showed strong, concurrent validity, diagnostic validity, and testretest reliability. We excluded participants with advanced Alzheimer's disease, because they were not difficult to identify; so, participants with mild-to-moderate Alzheimer's disease participated in this study, and the results of the PEACE test showed that Participants with Alzheimer's disease were significantly different from the control and MCI participants. The PEACE test differentiated between Participants with Alzheimer's disease and control participants at different educational levels, but it was unable to differentiate between literate and illiterate participants in the MCI group from those in the control group. While there was no difference between the illiterate and literate participants in the Alzheimer group, there was a significant difference between these two categories of participants in the control group and the MCI group. 
Memory loss is a natural part of aging. As people age, it is normal for them to have occasional memory problems, such as forgetting the name of a person they recently met. Memory loss that is not a normal part of aging is called dementia. Alzheimer's disease is the most common form of dementia (20).Lack of education is a major risk factor for developing dementia. The prevalence of dementia is about $16 \%$ among illiterates, while the percentage is $7 \%$ among literates. Elderly people with low levels of literacy have a steeper decline in both immediate and delayed recall of a list of words overtime than elderly people with higher literacy levels. Literacy skills are protective against declining memory among elderly people who do not have dementia (15). Literate individuals were postulated to possess a reserve factor that acted to delay or ameliorate the impairments of intellect and functioning that accompany neurodegenerative conditions (21). They could cope with advanced pathologic changes of the disease more effectively by maintaining function longer (22) and compensate for pathology through more efficient use of existing cognitive networks or recruitment of alternate networks (23-25). Due to lack of a logical relationship, we cannot claim that Alzheimer's disease is exacerbated by illiteracy; however, the results of the study do indicate that higher educational levels impede the adverse effects of Alzheimer's disease, which means that educational level modulates the scores on the PEACE test. In this study, literate and illiterate participants in the control group and in the MCI group were significantly different, and literate participants scored higher marks on the PEACE test, indicating that the PEACE assay was influenced by educational level. However, illiterate and literate participants in the Alzheimer's group did not get different scores on the PEACE test, indicating that the PEACE assay can be used with both literate and illiterate individuals. Diagnosing illiterate people in the absence of appropriate neuropsychological batteries for them presents major difficulties in conducting their neuropsychological evaluations, including misdiagnosis and/or delayed detection of dementia. Illiteracy and low levels of education are associated with both the increased risk of dementia and its misdiagnosis (6). So, the results of this study indicated that the PEACE instrument can make the challenge of correctly evaluating illiterate people somewhat less onerous.

We determined the cut-off point of the PEACE test for Alzheimer's participants. There was no significant difference between MCI participants and control participants, so we did not determine the cut-off point for the MCI group. Due to numerous questions in the PEACE test, this assay was more advantageous than others in which the difference between Participants with Alzheimer's disease and Participants without Alzheimer's disease is not obvious. The shortcomings of this study were its small sample size and the lack of an examination of the validity of the constructs for the sub-domains of the PEACE test. Also, the numerous questions in the PEACE test caused the participants to become tired.

\section{Conclusions}

There is a lack of validated instruments with adjustment of cut-points, translations, and replacements of culturespecific items. It is also difficult for professionals to communicate with people from different cultural backgrounds, especially if the client does not speak the same language. So, there is a risk of over-diagnosing dementia. The PEACE test addresses the limitations of existing tests and is appropriate for use in countries with a high illiteracy rate. It is a valid screening battery for detection of dementia in both illiterate and literate participants.

\section{Acknowledgments:}

The authors are grateful to the respected members of the research staff of Tehran University of Medical Sciences for their help with this study. The authors also express their gratitude to Dr. Maryam Noroozian (M.D., Professor of Neurology, Department of Geriatric Medicine, Tehran University of Medical Sciences, Tehran, Iran) for her help with the design of the PEACE assay design.

Funding: This research received no specific grant from any funding agency in the public, commercial, or not-forprofit sectors.

\section{Conflict of Interest:}

There is no conflict of interest to be declared.

\section{Authors' contributions:}

All authors contributed to this project and article equally. All authors read and approved the final manuscript. 


\section{References}

1) Fratiglioni L, Winblad B, von Strauss E. Prevention of Alzheimer's disease and dementia. Major findings from the Kungsholmen Project. Physiology \& behavior, 2007. 92(1):98-104. doi: 10.1016/j.physbeh.2007.05.059. PMID: 17588621

2) Prince M, Acosta D, Chiu H, Scazufca M, Varghese M. Dementia diagnosis in developing countries: a cross-cultural validation study. Lancet, 2003. 361(9361):909-17. doi: 10.1016/S0140-6736(03)12772-9. PMID: 12648969

3) Lucca U, Tettamanti M, Logroscino G, Tiraboschi P, Landi C, Sacco L, et al. Prevalence of dementia in the oldest old: The Monzino 80-plus population based study. Alzheimer's \& Dementia, 2015. 11(3):258-70. e3. doi: 10.1016/j.jalz.2014.05.1750. PMID: 25150732

4) Carcaillon L, Brailly-Tabard S, Ancelin ML, Tzourio C, Foubert-Samier A, Dartigues JF, et al. Low testosterone and the risk of dementia in elderly men: Impact of age and education. Alzheimer's \& Dementia, 2014. 10(5):S306-S314. doi: 10.1016/j.jalz.2013.06.006. PMID: 24035146

5) Jia J, Wang F, Wei C, Zhou A, Jia X, Li F, et al. The prevalence of dementia in urban and rural areas of China. Alzheimer's \& Dementia, 2014. 10(1):1-9. doi: 10.1016/j.jalz.2013.01.012. PMID: 23871765

6) Babacan-Yildiz G, Isika AT, Ur E, Aydemir E, Ertas C, Cebi M, Soysal P., et al. COST: Cognitive State Test, a brief screening battery for Alzheimer disease in illiterate and literate patients. International Psychogeriatrics, 2013. 25(03):403-12. doi: 10.1017/S1041610212001780. PMID: 23137551

7) Meng, X, D'Arcy C. Education and dementia in the context of the cognitive reserve hypothesis: a systematic review with meta-analyses and qualitative analyses. PLoS One, 2012. 7(6):e38268. doi: 10.1371/journal.pone.0038268. PMID: 22675535. PMCID: PMC3366926

8) Del-Ser T, Munoz DG, Hachinski V. An autopsy-verified study of the effect of education on degenerative dementia. Brain, 1999. 122(12):2309-19. doi: 10.1093/brain/122.12.2309 2309-2319. PMID: 10581224.

9) Ashford JW, Borson S, O'Hara R, Dash P, Frank L, Robert P., et al. Should older adults be screened for dementia? Alzheimer's \& Dementia, 2006. 2(2):76-85. doi: 10.1016/j.jalz.2006.02.005. PMID: 19595860

10) Caffarra P, Gardini S, Dieci F, Copelli S, Maset L, Concari L., et al. The qualitative scoring MMSE pentagon test (QSPT): a new method for differentiating dementia with Lewy Body from Alzheimer's disease. Behavioural neurology, 2013. 27(2):213-20. doi: 10.3233/BEN-120319. PMID: 23396218

11) Noroozian, M. The Elderly population in Iran: An ever growing concern in the health system. Iranian journal of psychiatry and behavioral sciences, 2012. 6(2):1. PMID: 24644476. PMCID: PMC3940007

12) Chandra V, Ganguli M, Ratcliff G, Pandav R, Sharma S, Belle S., et al. Practical issues in cognitive screening of elderly illiterate populations in developing countries. The Indo-US Cross-National Dementia Epidemiology Study. Aging Clinical and Experimental Research, 1998. 10(5):349-57. doi: 10.1007/BF03339881. PMID: 9932138

13) Park S, Park SE, Kim MJ, Jung HY, Choi JS, Park KH., et al. Development and validation of the Pictorial cognitive screening inventory for illiterate people with dementia. Neuropsychiatric disease and treatment, 2014. 10:1837. doi: 10.2147/NDT.S64151. PMID: 25285007. PMCID: PMC4181741

14) Cordell CB, Borson S, Boustani M, Chodosh J, Reuben D, Verghese J., et al. Alzheimer's Association recommendations for operationalizing the detection of cognitive impairment during the Medicare Annual Wellness Visit in a primary care setting. Alzheimer's \& Dementia, 2013. 9(2):141-150. doi: 10.1016/j.jalz.2012.09.011. PMID: 23265826

15) Noroozian, M, Shakiba A, Iran-Nejad S. The impact of illiteracy on the assessment of cognition and dementia: a critical issue in the developing countries. International Psychogeriatrics, 2014. 26(12):2051 2060. doi: 10.1017/S1041610214001707. PMID: 25166718

16) Sheehan, B. Assessment scales in dementia. Therapeutic advances in neurological disorders, 2012:1756285612455733. doi: 10.1177/1756285612455733. PMID: 23139705. PMCID: PMC3487532

17) Association, A.P. and A.A.o.F. Physicians, Diagnostic and statistical manual of mental disorders: Primary care version. 1996: American Psychiatric Pub.

18) McKhann G, Drachman D, Folstein M, Katzman R, Price D, Stadlan EM., et al. Clinical diagnosis of Alzheimer's disease Report of the NINCDS - ADRDA Work Group* under the auspices of Department of Health and Human Services Task Force on Alzheimer's Disease. Neurology, 1984. 34(7):939-44. PMID: 6610841

19) Abdollahpour I, Noroozian M, Nedjat S, Majdzadeh R. Caregiver burden and its determinants among the family members of patients with dementia in Iran. International journal of preventive medicine, 2012. 3(8):544. PMID: 22973484. PMCID: PMC3429801 
20) Jones RN, Fong TG, Metzger E, Tulebaev S, Yang FM, Alsop DC., et al. Aging, brain disease, and reserve: implications for delirium. The American Journal of Geriatric Psychiatry, 2010. 18(2):117-27. doi: 10.1097/JGP.0b013e3181b972e8. PMCID: PMC2848522

21) Qiu C, Bäckman L, Winblad B, Agüero-Torres H, Fratiglioni L. The influence of education on clinically diagnosed dementia incidence and mortality data from the Kungsholmen Project. Archives of Neurology, 2001. 58(12):2034-9. doi:10.1001/archneur.58.12.2034. PMID: 11735777

22) Manly JJ, Touradji P, Tang MX, Stern Y. Literacy and memory decline among ethnically diverse elders. Journal of clinical and experimental neuropsychology, 2003. 25(5):680-90. doi: 10.1076/jcen.25.5.680.14579. PMID: 12815505

23) Shao Z, Janes E, Visser K, Meyer AS. What do verbal fluency tasks measure? Predictors of verbal fluency performance in older adults. Frontiers in psychology, 2014. 5:772. doi: 10.3389/fpsyg.2014.00772. PMCID: PMC4106453

24) Caramelli P, Carthery-Goulart MT, Porto CS, Charchat-Fichman H, Nitrini R. Category fluency as a screening test for Alzheimer disease in illiterate and literate patients. Alzheimer Disease \& Associated Disorders, 2007. 21(1):65-7. doi: 10.1097/WAD.0b013e31802f244f. PMID: 17334275

25) Giffard B, Desgranges B, Nore-Mary F, Lalevée C, de la Sayette V, Pasquier F. The nature of semantic memory deficits in Alzheimer's disease. Brain, 2001. 124(8):1522-32. doi: 10.1093/brain/124.8.1522. PMID: 11459744 